\title{
First prototype of a silicon tracker using an 'artificial retina' for fast track finding
}

\author{
N. Neri*, A. Abba, F. Caponio, M. Citterio, S. Coelli, J. Fu, A. Geraci, M. Monti, \\ M. Petruzzo \\ INFN, Sezione di Milano, and Politecnico di Milano (Italy) \\ E-mail: nicola.neri@mi.infn.it
}

\section{F. Bedeschi, P. Marino, M. J. Morello, A. Piucci, G. Punzi, F. Spinella, S. Stracka, J. Walsh \\ INFN, Sezione di Pisa, Università di Pisa, and Scuola Normale Superiore di Pisa (Italy)}

\section{Ristori}

INFN, Sezione di Pisa (Italy), and Fermilab (USA)

D. Tonelli

\section{CERN (Switzerland)}

\begin{abstract}
We report on the R\&D for a first prototype of a silicon tracker based on an alternative approach for fast track finding. The working principle is inspired from neurobiology, in particular by the processing of visual images by the brain as it happens in nature. It is based on extensive parallelisation of data distribution and pattern recognition. In this work we present the design of a practical device that consists of a telescope based on single-sided silicon detectors; we describe the data acquisition system and the implementation of the track finding algorithms using available digital logic of commercial FPGA devices. Tracking performance and trigger capabilities of the device are discussed along with perspectives for future applications.
\end{abstract}

Technology and Instrumentation in Particle Physics 2014,

2-6 June, 2014

Amsterdam, the Netherlands

\footnotetext{
* Speaker.
} 


\section{Introduction}

The low level trigger capabilities of tracking systems have been proved to be crucial for the success of high energy physics experiments, especially at hadron colliders. The "artificial retina" is an approach for fast track finding based on an algorithm [1] that takes inspiration from neurobiology, in particular by the fact that specific neurons of the retina are specialised to identify specific shapes. In the case of the "artificial retina" we construct a grid of receptors that are tuned to identify charged particle tracks with different parameters. In this work we present the design of the first prototype of a tracking system with "artificial retina" capable of fast track finding with a latency $<1 \mu$ s and with track parameter resolutions that are comparable with the offline results. The prototype detector has an active area of about $100 \mathrm{~cm}^{2}$ and the maximal rate for track reconstruction is about $1 \mathrm{MHz}$. However, the "artificial retina" is a modular system that can be designed to work for HEP applications, i.e. high rates and large detectors, providing offline-like track quality results with a sub- $\mu$ s latency $[2,3,4]$.

\section{The Retina Algorithm}

For simplicity's sake, let's consider the reconstruction of a charged particle track in the $2 \mathrm{D}$ space $(x, z)$ using a tracking system composed of multiple layers. If $\left(x_{f}, z_{f}\right)$ and $\left(x_{l}, z_{l}\right)$ are the coordinates of a cluster on the first and the last tracking detectors, respectively, and $x_{ \pm}=\left(x_{f} \pm x_{l}\right) / 2$ and $z_{ \pm}=\left(z_{f} \pm z_{l}\right) / 2$, we can define the equation of a 2D track as

$$
x(z)=x_{+}+x_{-}\left(z-z_{+}\right) / z_{-} .
$$

The grid of track parameters $\left(x_{-}, x_{+}\right)$is shown in Fig. 1, where a cellular unit at position $i j$ corresponds to track receptors representing the intercepts of the ideal track with the detectors. A Gaussian receptor field is used to evaluate the goodness of the match of a set of clusters with a specific track hypothesis and a weight, $W_{i j}$, for each cell is calculated as

$$
\begin{array}{ll}
W_{i j}=\sum_{k} \exp \left(-\frac{s_{i j k}^{2}}{2 \sigma^{2}}\right) & \text { if } \quad s_{i j k}<2 \sigma, \\
W_{i j}=0 & \text { if } \quad s_{i j k}>2 \sigma,
\end{array}
$$

where $s_{i j k}=\left|x-x_{j,+}-x_{i,-}\left(z_{k}-z_{+}\right) / z_{-}\right|$is the distance of the cluster from the intercept of the track, associated with that cell, in layer $k$. The width of the receptor response field, $\sigma$, is much larger that the obtainable resolution on the track parameters and has to be adjusted for optimal response. A reasonable choice is $\sigma \simeq \Delta$, where $\Delta$ is the granularity of the grid of track parameters. A cluster $(x, z)$ is represented in the $\left(x_{-}, x_{+}\right)$plane as a line,

$$
x_{+}=-x_{-} \frac{z-z_{+}}{z_{-}}+x \text {. }
$$

The cluster information is sent in parallel to appropriate cellular units that calculate the weights. A matched track would result in a local maximum of the retina response $W_{i j}$, as reported in Fig. 2. 


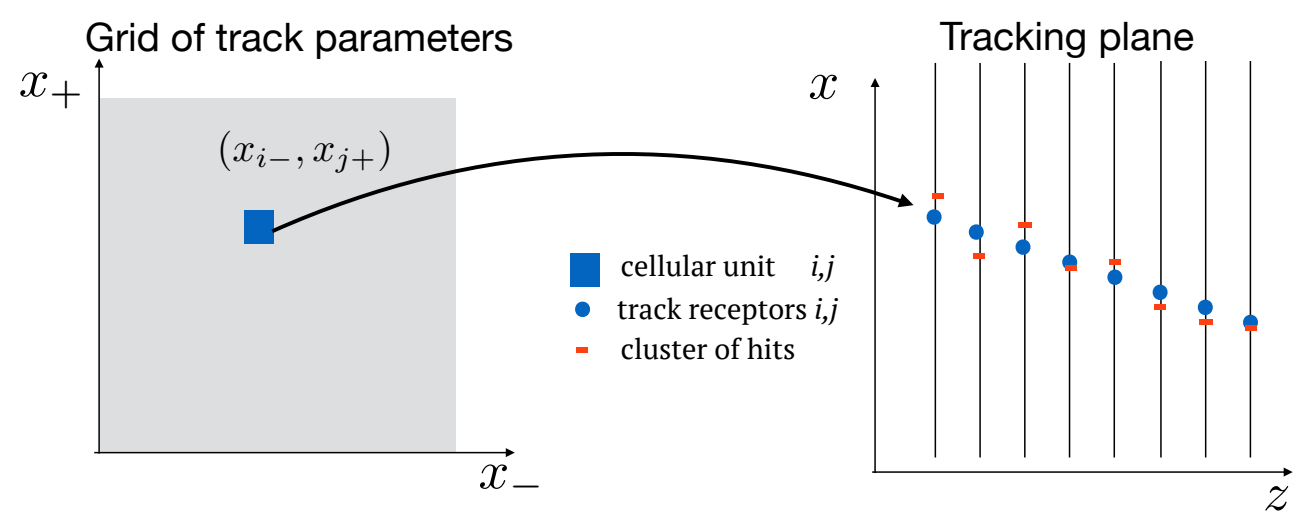

Figure 1: A cellular unit in the grid of parameters $\left(x_{-}, x_{+}\right)$identifies a specific track hypothesis. The corresponding track receptors represent the intercepts of the ideal track with the tracking layers. Cluster of hits corresponding to matched tracks are aligned with the track receptors within the experimental resolution.

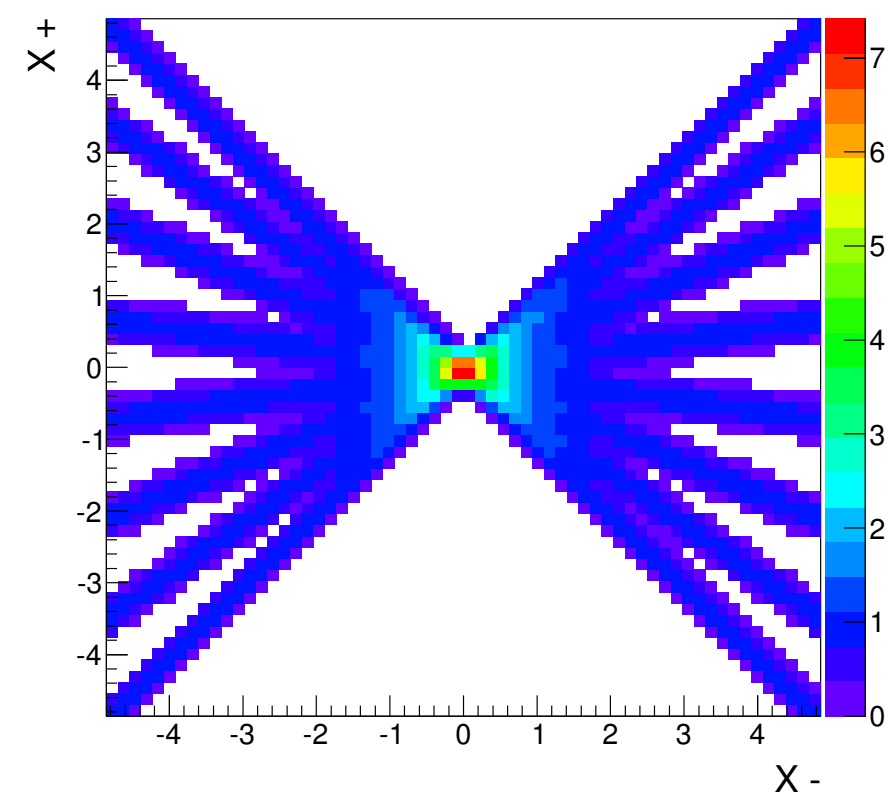

Figure 2: Simulation of the "artificial retina" response for the prototype silicon tracker with 8 layers. The intensity of the response (weight) is indicated by the colour code reported on the right. A reconstructed track is identified by a local maximum in the track parameter space.

\section{Tracking System Prototype with "Artificial Retina"}

The prototype system consists of 8 planes of single-sided silicon sensors with 512 strips each and $183 \mu \mathrm{m}$ pitch; the active area of the sensor is about $100 \mathrm{~cm}^{2}$ with $500 \mu \mathrm{m}$ thickness. The telescope layout is shown in Fig. 3. The sensors are arranged at a distance of $0.8 \mathrm{~cm}$ from each other and plastic scintillators are positioned at the top and the bottom of the telescope providing trigger signals for charged particle tracks traversing the tracking volume. 


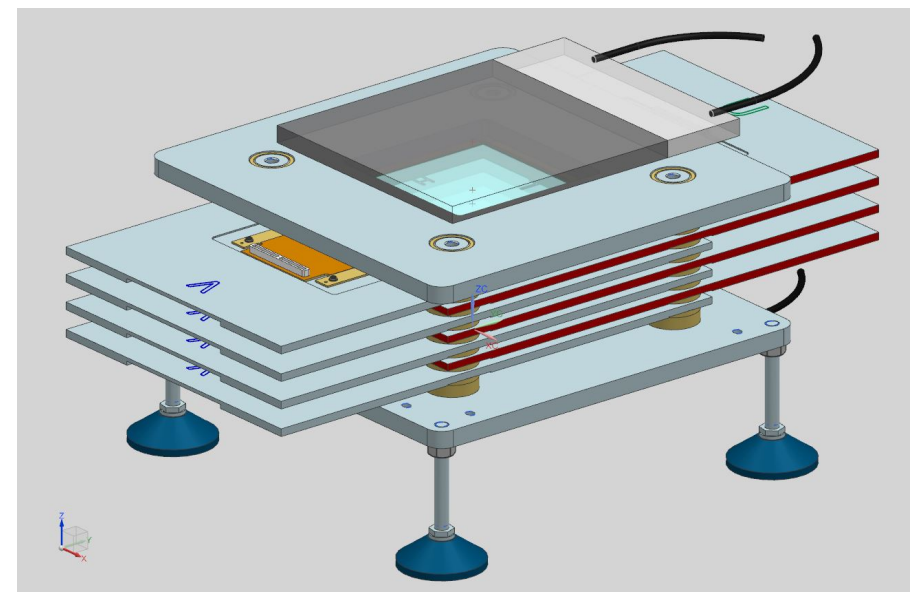

Figure 3: Telescope layout. The telescope consists of 8 planes of single-sided silicon sensors. Plastic scintillators are placed on the top and the bottom of the telescope providing external trigger for charged particle tracks traversing the tracking volume.

The expected rate for cosmic rays at sea level traversing all the telescope layers is of the order of $1 \mathrm{~Hz}$. The test with cosmic rays allows to verify all the functionalities of the system and to prove the working principle. The sensors are readout by a custom ASIC [5] which can accept trigger rates up to $1.1 \mathrm{MHz}$ and eventually a test with beam is envisaged to verify the behaviour at higher rates.

The schematic of the telescope readout system is shown in Fig. 4. It consists of a custom data acquisition (DAQ) board described in detail in Ref. [7]; here we report a short description of it. The ASIC provides the measurement of the hit position and the pulse height of 128 channels; each detector is readout by 4 ASICs and each one multiplexes 32 channels on a single analog output, for a total of 16 analog output channels for the readout of a plane of the telescope. The DAQ boards are based on FPGAs Xilinx Kintex 7 1x160, each one connected to 4 ADCs with 8 serial outputs at 80 mega-samples per second; they manage the readout ASICs and the sampling of the analog channels. The readout is performed at $40 \mathrm{MHz}$ on 4 channels for each ASIC that corresponds to a decoding of the telescope information at $1.1 \mathrm{MHz}$.

A second stage of the signal processing, for the calculation of the retina response, resides in the TEL62 board [6]. The "artificial retina" has been implemented using commercial FPGAs and its design is based on three main logic modules: a switch for the detector hits, a pool of engines for the digital processing of the hits, and a block for the calculation of the track parameters. The TEL62 board is equipped with 4 Altera Stratix III FPGAs that provide adequate computing performance for the switch, the engines, and the interpolation for determining the track parameters. The parameters of the tracks detected are finally transferred to host PC via Ethernet lane at $4 \mathrm{Gbit} / \mathrm{s}$. The FPGA resources of the TEL62 boards have been divided among the different modules: approximately 13\% for the switch module, $50 \%$ for the pool of engines and $12 \%$ for the track parameter determination. The rest is kept for backup. This configuration allows to realise an "artificial retina" with about 2000 cells with a clock frequency of the system of about $200 \mathrm{MHz}$. In general the number of cells depends on the requested precision on track parameters and their range. The number of cells scales linearly with the FPGA resources and the system is modular. The latency of the "artificial retina" response is determined by the number of clock cycles necessary for the hits to pass through the 
entire system, which is estimated to be less than 100. At a clock frequency of about $200 \mathrm{MHz}$ the latency is below $1 \mu \mathrm{s}$ and the system would allow for fast trigger decisions based on real time track parameter determination. The effect of the occupancy on the latency of the retina response is negligible for track rates below $1 \mathrm{MHz}$ and the latency is constant.

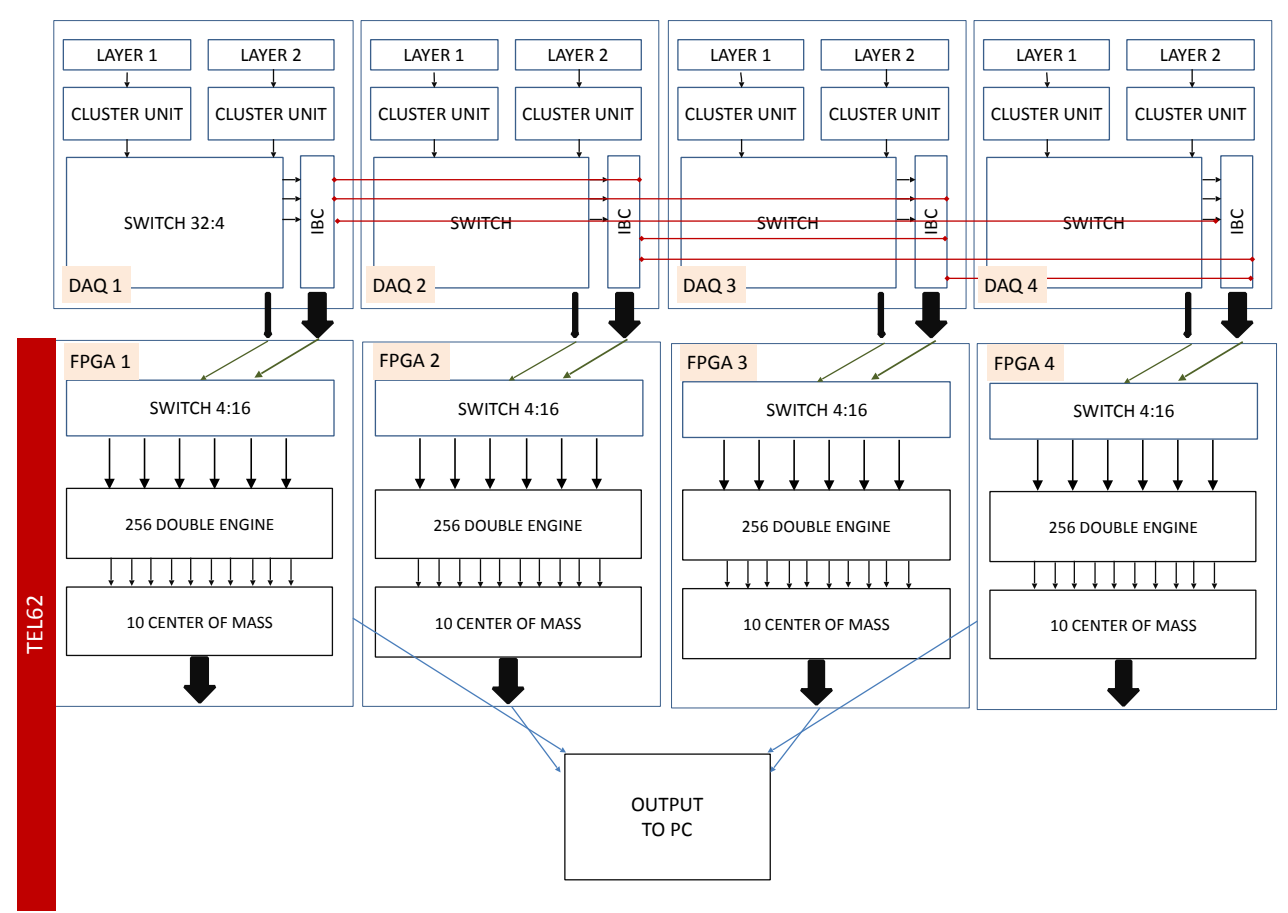

Figure 4: Schematics of the telescope readout system and of the retina architecture based on a TEL62 board.

\subsection{The Switch Module}

The grid of the retina parameters is divided in 4 regions corresponding to the number of available FPGAs in the TEL62 board. The switch module distributes in parallel the hits from all the 8 detector layers to appropriate logical units, called engines, which determine the good match (weight) of the hit combinations with a specific track hypothesis. A cluster is represented as a line in the space of parameters,

$$
\left|x-x_{+}-x_{-} \frac{z-z_{+}}{z_{-}}\right|<2 \sigma
$$

and its information has to be delivered to the engines belonging to the regions defined by Eq. 3.1, that reside in different FPGAs. The path of each hit is determined according to the cluster coordinates by a set of look-up tables (LUTs) and it is finally distributed to a set of engines corresponding to track parameters that are compatible with that specific hit. A graphical representation of the switch module is shown in Fig. 4, which is organised in two levels. The first level of the switch is placed in the Kintex-7 FPGA of the readout board and distributes data from 32 inputs to 4 outputs. The 8 layer telescope is readout using 4 DAQ boards: each readout board receives signals from 2 detector planes on 32 analog inputs. The switch module sends the signals on 4 output ports: one 
output port is connected to the second level of the switch in the TEL62 board, and the other output ports are connected to the other three readout boards for a fully meshed network. The second level of the switch resides in the FPGAs of the TEL62 board and distributes the hits from 4 inputs to 16 outputs, each one delivering the data in parallel to 16 engines. The switch module relies on a basic $16 \times 16$ way data dispatcher where only specific input and output ports are connected. Each engine calculates the weight for 2 cellular units for a total of 2048 cellular units for the retina.

\subsection{The Engine Module}

The engine corresponds to a specific cell at position $i j$ in the grid of the track parameter space $\left(x_{-}, x_{+}\right)$and its excitation is proportional to the weight, $W_{i j}$, defined in Eq. 4.1. In Fig. 5

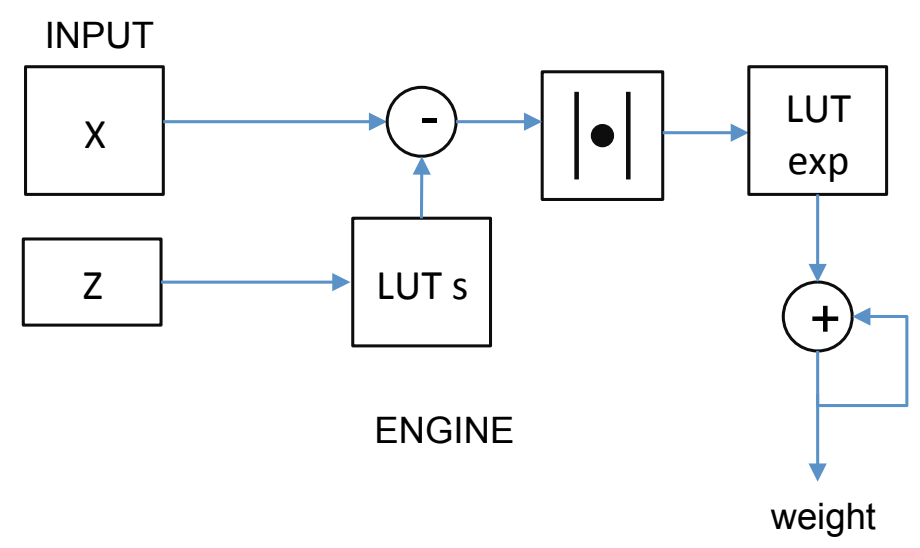

Figure 5: Diagram of the logical blocks of the engine. The distance between the cluster with coordinates $(x, z)$ from the track intercept in a specific layer is calculated using a LUT. Another LUT is used for the evaluation of the exponential function of the weight.

it is shown the diagram of the logical blocks of the engine where two LUTs with 1024 16-bit integers have been used for the calculation of the weights. One LUT is used for the calculation of the distance $s_{i j k}$ and the other LUT is used for the calculation of the exponential function. Each engine calculates the weights for two neighbouring cells in the retina of the track parameters. Each engine interacts with the nearest neighbour engines to determine the maximal weight value and then another logical block interpolates the track parameters from other two adjacent engines in the appropriate directions.

\subsection{The Track Module}

The engine determines if the calculated weight corresponds to a local maximum and then sends its weight, and the ones from the adjacent cells, to the track module. The track parameters are determined by Gaussian interpolation of the weights of neighbouring cells

$$
x=x_{0}+\frac{\Delta}{2} \frac{\log \left(W_{-} / W_{0}\right)-\log \left(W_{+} / W_{0}\right)}{\log \left(W_{-} / W_{0}\right)+\log \left(W_{+} / W_{0}\right)}
$$

where the subscript 0 identifies the local maximum, and,+- identify the adjacent cells. The values of the $\log (x)$ function are stored in a $10 \times 16$ bit LUT. In the simulation we used a grid step 
$\Delta=1.6 \mathrm{~mm}$ which is much larger with respect to the strip pitch of the sensors of $183 \mu \mathrm{m}$. The resolutions on the track parameters $x_{+}$and $x_{-}$calculated with the "artificial retina" were found to be comparable with the offline results obtained with a $\chi^{2}$ fit of the clusters to a straight line, as shown in Fig. 6. The resolution on the $x_{+}$parameter is about $20 \mu \mathrm{m}$ for offline reconstructed tracks and for tracks reconstructed with the artificial retina. For the $x_{-}$parameter we obtain a resolution of $27 \mu \mathrm{m}$ and $31 \mu \mathrm{m}$ with the offline and the artificial retina reconstruction, respectively. Similar
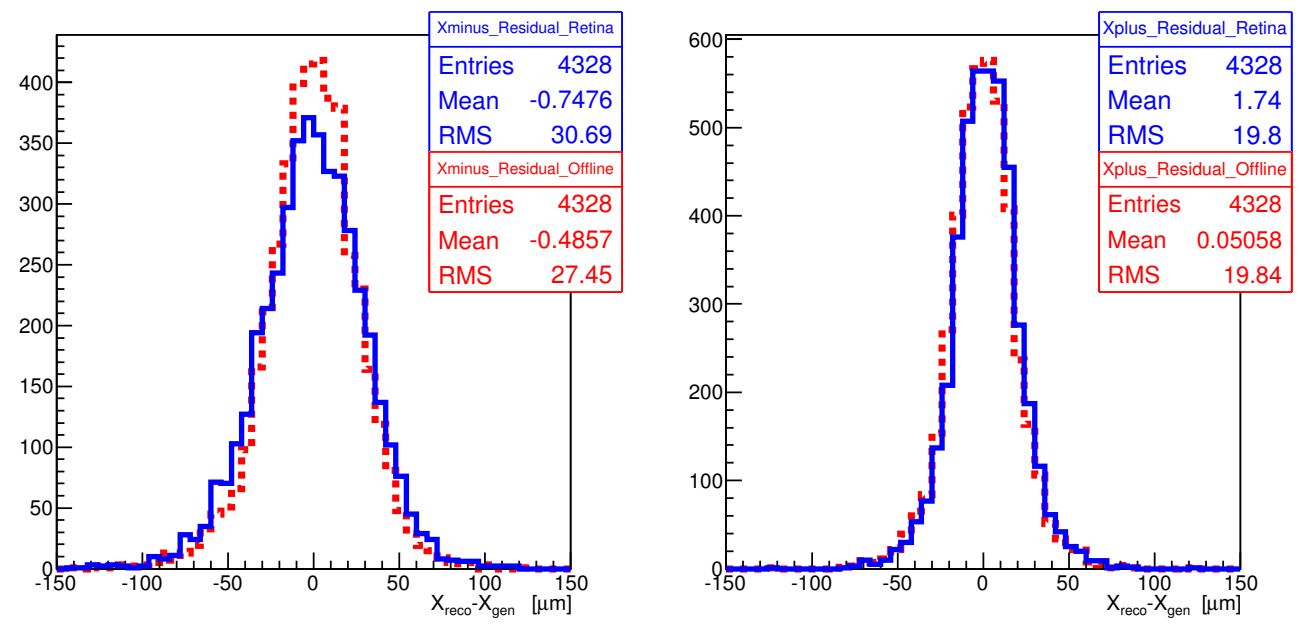

Figure 6: Resolution for $x_{-}$(left) and for $x_{+}$(right) track parameters obtained with the "artificial retina" (open blue histogram) compared with offline results (dashed red histogram).

results have been obtained with a center-of-mass calculation,

$$
x=\frac{\sum_{i=-, 0,+} W_{i} x_{i}}{\sum_{i=-, 0,+} W_{i}}
$$

and then applying a correction for the bias, $\delta x=\alpha\left(x-x_{0}\right)$, where $\alpha$ is a constant.

\section{Perspectives for future applications: time-tagged events}

Recent advances in silicon detectors make attractive the possibility to include the precise time information of the hit in track reconstruction algorithms. The gigatracker silicon detector prototypes for the NA62 experiment achieved sub-ns time resolution [10], and recent R\&D activities on ultra fast silicon detectors aim to achieve time resolution below $20 \mathrm{ps}$ in the near future [11]. The precise time information of the hit could be used to improve the retina performance. In this case, the weight can be defined as a function of the time of the track at the origin, $t$, as

$$
W_{i j}(t)=\sum_{k} \exp \left(-\frac{s_{i j k}^{2}}{2 \sigma^{2}}\right) \exp \left(-\frac{t_{i j k}^{2}}{2 \sigma_{t}^{2}}\right),
$$

where $t_{i j k}=\left|t_{k}-\left(t+\Delta t_{i j k}\right)\right|$ and $t_{k}$ is the time of the hit at layer $k, t$ is the time of the track at the origin, and $\Delta t_{i j k}$ is the estimated time for a particle, whose trajectory corresponds to the retina cell 
$i j$, to arrive at layer $k$. Noise hits out of time are suppressed by the corresponding weights where $\sigma_{t}$ is proportional to the resolution on the time of the hit and can be adjusted for optimal response; in Fig. 7 it is shown the retina response in presence of background hits with no time information (left) and with time information (right). The plots correspond to a simulation with detector occupancy of $1 \%$ and resolution on the time of the hit of $100 \mathrm{ps}$. In particular, the time $t$ can be determined from interpolating weights at different times. If the time is divided in 3 intervals around the nominal time of the track, which is supposed to be known with a relative large uncertainty, then the time of the track at the origin can be determined with a precision $\sim \sigma_{h} / \sqrt{N}$, where $\sigma_{h}$ is the resolution on the time of the hit and $N$ is the number of tracking detectors. As an example, the time of the track $t$ can be determined with a precision of about 30 ps with 8 tracking detectors with 100 ps hit time resolution.
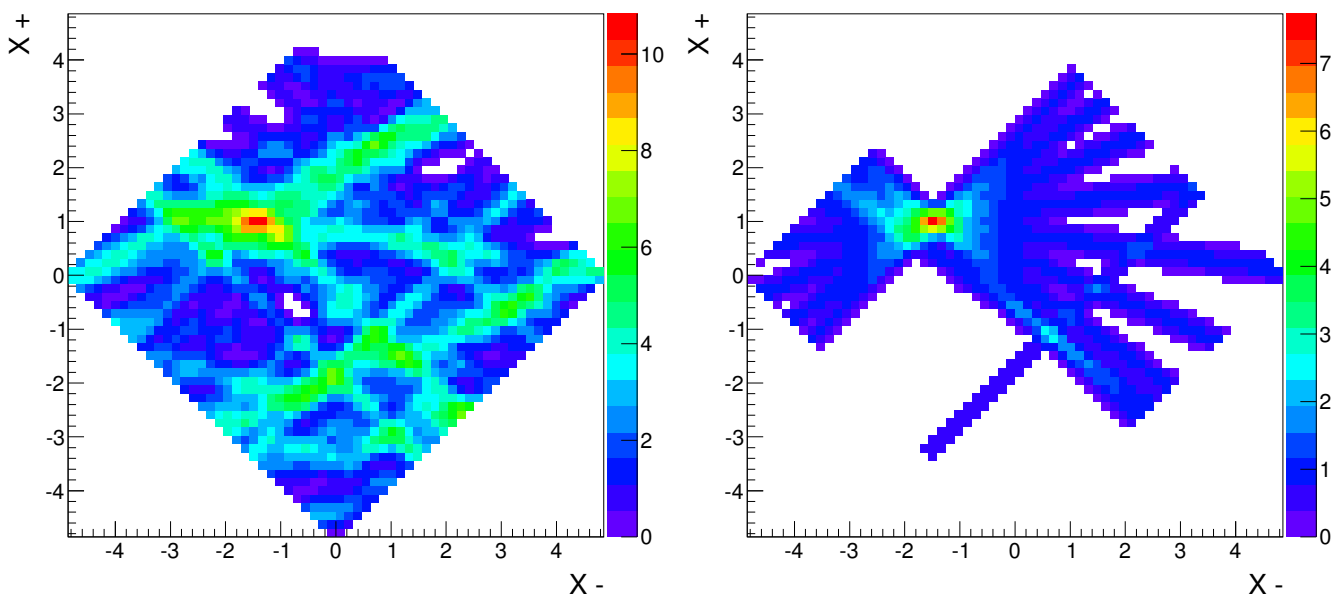

Figure 7: The retina response in presence of background hits with no time information (left) and with time information right (right).

\section{Conclusions}

The design of the first prototype of a tracking system with "artificial retina" has been presented. It consists of 8 planes of single-sided silicon strip detectors with custom ASIC readout where the "artificial retina" architecture is implemented using commercial FPGAs. The telescope can operate up to $1 \mathrm{MHz}$ track rate. The working principle can be proved using cosmic rays and eventually a test with a beam is envisaged to test the system at higher rates. The system is organised in three modules: a switch network for the parallel distribution of the hits, a block of engines for the calculation of the excitation of the cells of the retina and a module for the calculation of the track parameters. The tracking performance of the system are comparable with offline results with a latency of the response $<1 \mu$ s which allows fast trigger decisions based on track parameters. The system is modular and can be dimensioned for larger tracking detectors with high particle rates $[2,3,4]$.

An interesting perspective for future applications is represented by the use of precise information of the time of the hit with a resolution better than 100 ps. This would allow an improved 
performance of the retina in presence of background hits and also the determination of time-tagged tracks in real time.

\section{References}

[1] L. Ristori, “An artificial retina for fast track finding," Nucl. Instrum. Meth. A 453, 425 (2000).

[2] A. Abba, F. Bedeschi, M. Citterio, F. Caponio et al., "A specialised track processor for the LHCb upgrade,” LHCb note, CERN-LHCb-PUB-2014-026 (2014).

[3] G. Punzi et al., "A Specialized Processor for Track Reconstruction at the LHC Crossing Rate", Proceedings of INSTR14 conference, BINP, Novosibirsk (2014).

[4] D. Tonelli et al., "A Specialised Processor for Track Reconstruction at the LHC Crossing Rate", Proceedings of TIPP2014 conference, Amsterdam, The Netherlands (2014).

[5] S. Löchner and M. Schmelling, "The Beetle Reference Manual - chip version 1.3, 1.4 and 1.5", LHCb-2005-105 (2006).

[6] B. Angelucci, E. Pedreschi, M. Sozzi and F. Spinella, "TEL62: an integrated trigger and data acquisition board,” IEEE Nucl. Sci. Symp. Conf. Rec. 2011, 823 (2011).

[7] F. Caponio, A. Abba, N. Lusardi, A. Geraci et al., "The Readout Architecture for the Retina-Based Cosmic Ray Telescope," Conference Record of 19th Real-Time Conference, Nara, Japan (2014).

[8] LHCb Collaboration, “LHCb VELO Upgrade Technical Design Report," Technical Design Report LHCb, CERN-LHCC-2013-021 (2013).

[9] LHCb Collaboration, “LHCb Tracker Upgrade Technical Design Report,” Technical Design Report LHCb, CERN-LHCC-2014-001 (2014).

[10] S. Garbolino, G. Aglieri Rinella, V. Carassiti, A. Ceccucci, E. Cortina, J. Daguin, G. Dellacasa and M. Fiorini et al., "Results from the gigatracker prototypes: Two pixel front-end ASICs with sub-ns time resolution for the NA62 experiment," PoS RD 11, 033 (2011).

[11] N. Cartiglia, M. Baselga, G. Dellacasa, S. Ely, V. Fadeyev, Z. Galloway, S. Garbolino and F. Marchetto et al., "Performance of Ultra-Fast Silicon Detectors," JINST 9, C02001 (2014). 